\title{
Energy Collectives: a Community and Fairness based Approach to Future Electricity Markets
}

\author{
Moret, Fabio; Pinson, Pierre
}

Published in:

IEEE Transactions on Power Systems

Link to article, DOI:

10.1109/TPWRS.2018.2808961

Publication date:

2019

Document Version

Peer reviewed version

Link back to DTU Orbit

Citation (APA):

Moret, F., \& Pinson, P. (2019). Energy Collectives: a Community and Fairness based Approach to Future Electricity Markets. IEEE Transactions on Power Systems, 34(5), 3994-4004.

https://doi.org/10.1109/TPWRS.2018.2808961

\section{General rights}

Copyright and moral rights for the publications made accessible in the public portal are retained by the authors and/or other copyright owners and it is a condition of accessing publications that users recognise and abide by the legal requirements associated with these rights.

- Users may download and print one copy of any publication from the public portal for the purpose of private study or research.

- You may not further distribute the material or use it for any profit-making activity or commercial gain

- You may freely distribute the URL identifying the publication in the public portal

If you believe that this document breaches copyright please contact us providing details, and we will remove access to the work immediately and investigate your claim. 


\title{
Energy Collectives: a Community and Fairness based Approach to Future Electricity Markets
}

\author{
Fabio Moret, Pierre Pinson, Senior Member, IEEE
}

\begin{abstract}
While power system organization has evolved from a hierarchical structure to a more decentralized model, electricity markets are still not up to date with the ongoing transformation towards more consumer-centric economies. As Information and Communication Technologies (ICT) are broadly adopted, they allow prosumers to have a more proactive role in power system operation. This work introduces the concept of energy collectives, as a community-based electricity market structure. We find that when prosumers are allowed to share energy at community level, overall electricity procurement for the community reflects prosumers' preferences. We show that community members can be influenced by a supervisory third-party in charge of interfacing with the market and system operator and of guaranteeing the collective common agreements. We simulate a number of test cases and we apply typical principles from analysis of communication networks and distributed systems to assess community fairness.
\end{abstract}

Index Terms-Electricity markets, prosumers community, renewable energy integration, distributed optimization, fairness.

\section{NOMENCLATURE}

Only the most important symbols of this paper are included.

$\alpha_{j} \quad$ Energy imported by prosumer $j$.

$\beta_{j} \quad$ Energy exported by prosumer $j$.

$\delta_{j} \quad$ Upper bound of $\alpha_{j}$.

$\gamma \quad$ Penalization coefficient (the subscript identifies the specific application).

$\lambda_{j} \quad$ Price of energy perceived by prosumer $j$.

$\lambda_{\text {DA }} \quad$ Day-ahead market price.

$\mathscr{P}_{j} \quad$ Feasible space of $p_{j}$.

$\mathscr{R}_{j} \quad$ Feasible space of prosumer $j$.

$\mathscr{U}_{i j} \quad$ Feasible space of $u_{i j}$.

$\bar{U}_{i j} \quad$ Upper bound of asset $i$ of prosumer $j$.

$\psi_{i j} \quad$ Cost/utility function of asset $i$ of prosumer $j$.

$\rho \quad$ Penalization factor of $L_{\rho}$.

$\tau \quad$ Price spread between import and export price.

$\tilde{f}_{j} \quad$ Extended cost function of prosumer $j$.

$\underline{U}_{i j} \quad$ Lower bound of asset $i$ of prosumer $j$.

$c_{i j} \quad$ Quadratic coefficient of $\psi_{i j}$.

$d_{i j} \quad$ Linear coefficient of $\psi_{i j}$.

$f_{j} \quad$ Cost function of prosumer $j$.

$g \quad$ Model of community manager.

$h_{j} \quad$ Preferences and/or transaction costs of prosumer $j$.

$I_{j} \quad$ Set of assets of prosumer $j$.

$L_{\rho} \quad$ Augmented Lagrangian.

$n \quad$ Number of prosumers in the collective.

$N_{j} \quad$ Set of members of neighbourhood $j$.

F. Moret and P. Pinson are with the Technical University of Denmark, Department of Electrical Engineering, Kgs. Lyngby, Denmark (emails: \{fmoret,ppin\}@elektro.dtu.dk). The work is partly supported by the Danish ForskEL programme through the Energy Collective project (grant no. 20161-12530). $p_{j} \quad$ Net energy produced by prosumer $j$

$q_{j} \quad$ Energy imported by prosumer $j$ from the community.

$q_{\text {exp }} \quad$ Total energy exported.

$q_{\text {imp }} \quad$ Total energy imported.

$r_{k} \quad$ Vector of primal residuals.

$s_{k} \quad$ Vector of dual residuals.

$u_{i j} \quad$ Power set-point of asset $i$ of prosumer $j$.

$v_{i j} \quad$ Energy traded between neighbourhood $i$ and $j$.

$y \quad$ Vector of Lagrangian multipliers.

\section{INTRODUCTION}

$\mathbf{T}$ HE MASSIVE DEPLOYMENT of renewable generation challenges common practice in power system operation, not only in the form of large scale power plants, but also of Distributed Energy Resources (DERs). Simplifying DERs as load reduction for electricity consumers may have been acceptable at low penetration levels. However, as their installed capacity rises, DERs enhance end-users flexibility. If appropriately controlled, this flexibility can reveal strategic opportunities for supply and demand at distribution level [1].

Increasing awareness of their energy consumption allow consumers to go beyond common energy management (e.g. energy efficiency and time-varying electricity tariffs), and towards a more proactive role in the power system. Demand Side Management (DSM) benefits have been largely investigated in the past [2]. However, to provide services to the power system, for instance ancillary services or peak-shaving, DERs are normally considered as a group, increasing the total available flexibility. As Information and Communication Technologies (ICT) are broadly employed in energy systems, it becomes more likely to optimally coordinate energy resources in a distributed fashion. Common solutions include control architectures such as micro-grids management, virtual power plants and aggregators, as largely reviewed in [3], [4]. When optimized through control architectures, end-users respond to control signals issued by a central agent, that seeks profit maximization. However, individual preferences and social perspectives justify the need for prosumer-centric market mechanisms, in which prosumers actively trade their energy according to their individual objectives and not those of a third party [5].

Future prosumer markets may envisage more or less structured organizations depending on the degree of decentralization considered [6]. A fully decentralized market conformation yields peer-to-peer trades among all participants with no need for third-party supervision [7], [8]. On the contrary in a distributed structure, all peers communicate with a central node that supervises the negotiation process. Technological solutions exist to enable these market organizations, for instance 
on the cloud for distributed systems [9] or using a blockchain for decentralized mechanisms [10].

In view of a consumer-centric market, the presence of a supervisory node simplifies market regulation and the interface with the market and system operator. For this reason, we believe that community-based distributed structures will more likely develop in the near future. We call this market organization energy collective and we define it as a community of prosumers that operates in a collaborative manner, optimizing usage of resources. This translates to a market framework where the collective members can trade their lack or excess of energy. The distributed nature of the market implies that all prosumers are in charge of optimizing their assets individually. Optimality is achieved as prosumers are coordinated by a nonprofit virtual node, that we call community manager.

Building on the concepts of transactive energy [11] and energetic communities [12], communities of prosumers have been proposed, where groups of end-users can coordinate their energy utilization. Specifically, our proposal is to be placed into perspective with related research that recently appeared in the power system literature, e.g., [13]-[16]. We find that these models simplify the problem as the relation to market-based operation is not considered. In the proposed market structure, we include the interface with the market and system operator by means of the community manager. Our first key contribution is then to analyze how the decisions of the players are affected by market mechanisms, influenced by the community manager, and not controlled by control architectures. As we envisage the community manager also as a guarantor of the common goals within the community, our second key contribution consists in the assessment of fairness among market participants.

Compared to existing market mechanisms, the proposed energy collective reflects true preferences of consumers in the negotiation process. Moreover, privacy protection of preference and strategy schemes for each participant is granted while the computational burden is distributed among players. The generality of the negotiation mechanism formulation allows this market organization to accommodate different actors as well as different preference schemes. Transactive energy control systems or control architectures can therefore be included in an energy collective, by modelling virtual agents, e.g. aggregators or micro-grids management systems, as participants of the community.

The paper is organized as following. Section II introduces market organization for energy collectives as well as the modelling of the community members. In Section III, we present and discuss the fundamental role of the community manager. In Section IV we provide the methodology to characterize and simulate the community negotiation process based on distributed optimization concepts. Subsequently, we use a number of test cases in Section V to validate the proposed market structure as well as to verify the influence of community manager models on participants' behaviours. Finally, Section VI gathers conclusions and perspectives regarding future works based on energy collectives.

\section{ENERgy COLlectives AND THEIR OPERATIONAL FRAMEWORK}

The market structure for energy collectives is designed to provide a general framework easy to adapt to different contingent situations. We define the market independently of the nature of the prosumers assets. This implies that different DERs can be included without the need to modify the market mechanisms. The presence of a supervisory node facilitates the interface to different markets, as the collective members are represented by a single node. We consider that a community, especially of large size, can interface with different existing markets, e.g., wholesale, balancing, ancillary services, as well as future market designs. Figure 1 displays a possible future market organization with peer-to-peer transactions among communities and nested optimization mechanisms, where subcommunities become assets of higher level collectives. In case of small size communities, as the one considered as test case in this paper, the community manager can interface with retailers and their respective contracts. Note that there exist utilities offering the consumers to have a dynamic electricity tariff that, for instance, follows the trend of the day-ahead market price. In the proposed market organization, this is simply resolved by choosing difference import and export price series.

\section{A. Market organization}

From a market perspective, prosumers are defined only by their energy trades within and outside the community, while the community manager operates as interface between prosumers and two market layers. A market layer at community level allows the members of the collective to share their excess or lack of energy. A second layer envisages trades between the community, as a whole, and the market and system operator.

To better illustrate the market model, let us consider an energy collective composed by $n$ prosumers (indexed with $j=$ $1, \ldots, n)$, each of them with net energy production $p_{j}$ and total costs $f_{j}$. The optimal community dispatch is then obtained as the solution of the exchange problem

$$
\begin{array}{ll}
\min _{\Gamma} & \sum_{j=1}^{n} f_{j}\left(p_{j}, q_{j}, \alpha_{j}, \beta_{j}\right)+g\left(q_{\text {imp }}, q_{\text {exp }}, \vartheta\right) \\
\text { s.t. } & p_{j}+q_{j}+\alpha_{j}-\beta_{j}=0, \quad j=1, \ldots, n \\
& \sum_{j=1}^{n} q_{j}=0 \\
& \sum_{j=1}^{n} \alpha_{j}=q_{\text {imp }} \\
& \sum_{j=1}^{n} \beta_{j}=q_{\exp } \\
& p_{j} \in \mathscr{P}_{j}, \\
& \alpha_{j}, \beta_{j} \geq 0, \quad j=1, \ldots, n \\
& j=1, \ldots, n
\end{array}
$$

where $\Gamma=\left\{p_{j}, q_{j}, \alpha_{j}, \beta_{j}\right\}$ is the set of decision variables and $q_{\text {imp }}, q_{\text {exp }}$ are continuous variables describing import and export exchanges with the system operator. For each prosumer 


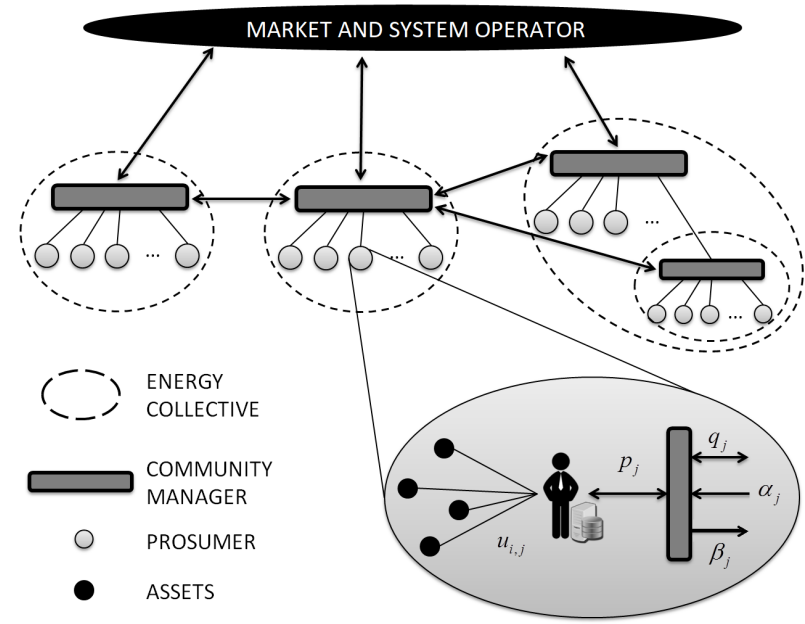

Fig. 1. Organization of an energy collective and its interactions with system operator and other collectives.

we consider technical constraints (1f) and power balance (1b) between net production, $p_{j}$, and energy traded. This energy can be traded at community level (1c) and with the system operator (1d)-(1e). Hence, we describe the energy traded by each prosumer as sum of the energy exchanged within the community, $q_{j}$, and the energy each prosumer has to respectively import, $\alpha_{j}$, or export, $\beta_{j}$, from outside the community, as in Figure 1.

The core of this market organization is the inclusion, in the negotiation process, of common agreements on how the energy collective is to jointly handle its internal objectives and its interaction with the outside world. We address this role to the community manager and we model it in (1) by means of the function $g$. We define $g$ only by global variables ( $q_{\text {imp }}$ and $q_{\exp }$ ) and by a set of relevant parameters or additionally variables $\vartheta$. Different community agreements can be included only by operating on the community manager, without modifying the prosumers model. Section III is reserved to the modelling and discussion of the fundamental role of this term in the operation of energy collectives.

\section{B. The prosumers model}

As we adopt a distributed structure, each prosumer $j$ is in charge of optimizing its set $I_{j}$ of assets (indexed with $i \in I_{j}$ ). For a given market time unit, each player has to find the optimal power set-points for each asset, $u_{i j}$ (positive for energy produced), in view of the respective cost function, $\psi_{i j}\left(u_{i j}\right)$ and the technology constraints, $\mathscr{U}_{i j}$. For the sake of this work, we assume quadratic costs and utility functions for each asset,

$$
\psi_{i, j}\left(u_{i j}\right)=c_{i j} u_{i j}^{2}+d_{i j} u_{i j}
$$

with $c_{i j} \geq 0$. However, all the considerations in this paper hold as long as the functions $\psi_{i j}$ are convex, preserving the convexity of the entire optimization problem and, hence, ensuring that the solution found coincides with the global optimum. Note that in case of non-convex problems decomposition techniques can be adjusted to reach optimality. However, as there is no guarantee of global but only local optimality, it is common procedure to apply out-of-market corrections to cope with suboptimality.

Since we aim for an assets independent formulation, prosumers are defined in the community market only by their net production $p_{j}=\sum_{i \in I_{j}} u_{i j}$. In order to decompose the global optimization problem into sub-problems specific for each prosumer $j$, we define $\tilde{f}_{j}$ as an extension of $f$ in (1), including all prosumer-specific information, as

$$
\begin{aligned}
\tilde{f}_{j}\left(p_{j}, q_{j}, \alpha_{j}, \beta_{j}\right)= & \sum_{i \in I_{j}} \psi_{i j}\left(u_{i j}\right)+h_{j}\left(q_{j}, \alpha_{j}, \beta_{j}\right) \\
& +I_{\mathscr{R}_{j}}\left(p_{j}, q_{j}, \alpha_{j}, \beta_{j}\right)
\end{aligned}
$$

We use the indicator function $I_{\mathscr{R}_{j}}\left(p_{j}, q_{j}, \alpha_{j}, \beta_{j}\right)$ on the set

$$
\begin{aligned}
\mathscr{R}_{j}= & \left\{\left(p_{j}, q_{j}, \alpha_{j}, \beta_{j}\right) \mid p_{j}=\sum_{i \in I_{j}} u_{i j}, u_{i j} \in \mathscr{U}_{i j} \text { for } i \in I_{j},\right. \\
& \left.p_{j}+q_{j}+\alpha_{j}-\beta_{j}=0, \alpha_{j} \geq 0, \beta_{j} \geq 0\right\}
\end{aligned}
$$

to include all the constraints related to the single prosumers $\left(\mathscr{P}_{j} \bigcap_{i \in I_{j}} \mathscr{U}_{i, j}\right)$ and the function $h$ to include homogeneous costs previously agreed within the community, e.g. transaction costs, as well as heterogeneous preference schemes. For instance, if we assume $h$ to be

$$
h_{j}\left(q_{j}, \alpha_{j}, \beta_{j}\right)=\gamma_{\mathrm{com}}\left|q_{j}\right|+\gamma_{\mathrm{imp}} \alpha_{j}+\gamma_{\mathrm{exp}} \beta_{j}
$$

the optimization of prosumer $j$, and hence the equilibrium of the whole collective, is influenced accordingly to the weighting coefficients. Favourable transaction costs for internal energy exchanges, $\gamma_{\text {com }}$, will encourage prosumer $j$ to trade more inside the community, while extra penalization for import and export of energy, $\left(\gamma_{\mathrm{imp}}, \gamma_{\mathrm{exp}}\right)$, may reflect the will of prosumer $j$ to increase its own autonomy from market and system operator.

\section{Market efficiency and incentive compatibility}

The proposed market design for energy collectives ensures, by construction, market efficiency. As demonstrated in the Appendix, the Karush-Kuhn-Tucker (KKT) conditions of the optimization problem, as in (1), correspond to those of an equilibrium problem in which each agent individually minimizes its costs. This implies that both problems achieve the same optimal solution under different assumptions. Perfect competition is assumed in the equilibrium problem solution, while in the central optimization problem every agent has to reveal its utility curves. However, by decomposing the optimization problem, we are able to limit the set of information needed to grant market efficiency, enhancing the privacy level of the participants utility and cost curves as well as their preferences.

While demonstrating market efficiency, the assumption of a perfect competitive market excludes the possibility of exercising market power within the community. To ensure incentive compatibility of the proposed market, one should analyze system equilibria under heterogeneous strategic behaviours, that could be modelled by means of function $h$, and check for possibility of exercising market power, e.g. by modelling the problem as a Stackelberg leader-follower game. As this implies 
an independent line of work and a significant contribution to the state-of-the-art, we leave these issues as future work. Therefore in this study, we assume homogeneous transaction costs and we consider that every participant can maximize its objective just by acting according to its true preferences.

\section{The Role of Community Manager}

As already mentioned, we envisage the community manager not only as supervisor of convergence to system optimality but also as interface between collective members and market and system operator. In theory, one can implement any decision model $g$, as long as it preserves convexity of the whole formulation. We encourage the reader to interpret the interface models proposed below as some of the several possible examples. Different definitions for $g$ are to impact the behaviour of its members, as well as their revenues and payments. This strongly links to existing work in literature focusing on congestion management of, e.g., telecommunication networks or electrical grids with charging of electric vehicles [17], where concepts such as proportional fairness and max-flow protocols are built upon.

\section{A. Market-based interface}

In the most simple market-driven case, the community tries to minimize the costs of importing and to maximize the revenues from exporting energy with the day-ahead market. The community interface can be modelled as

$$
g\left(q_{\mathrm{imp}}, q_{\mathrm{exp}}\right)=\left(\lambda_{\mathrm{DA}}+\tau\right) q_{\mathrm{imp}}-\lambda_{\mathrm{DA}} q_{\mathrm{exp}}
$$

where $\lambda_{\mathrm{DA}}$ is the wholesale market price. Depending on the contingent regulation, the price at which the community could buy energy from the power market is generally higher than the selling one. To consider this possibility, we include a parameter $\tau$ describing the spread between import and export price.

\section{B. Community autonomy}

In case the community wants to be as autonomous as possible, one will naturally have

$$
g\left(q_{\text {imp }}\right)=\gamma_{\text {imp }}\left\|q_{\text {imp }}\right\|_{l}+\gamma_{\text {exp }}\left\|q_{\exp }\right\|_{l}
$$

where $\|\cdot\|_{l}$ is the $l$-norm with $l=1, \ldots, \infty$ and $\gamma_{\text {imp }}$ and $\gamma_{\exp }$ the penalty factors. In case of a $l_{1}$-norm, (4) becomes

$$
g\left(q_{\text {imp }}, q_{\exp }\right)=\gamma_{\text {imp }} q_{\text {imp }}+\gamma_{\exp } q_{\exp }
$$

For $\gamma_{\mathrm{imp}}=\left(\lambda_{\mathrm{DA}}+\tau\right)$ and $\gamma_{e}=-\lambda_{\mathrm{DA}},(6)$ generalizes (4), with $\gamma_{\mathrm{imp}}$ and $\gamma_{\text {exp }}$ representing the price the community is willing to pay (when positive, or to receive as compensation when negative) for exchanging energy with the market or system operator.

As we analyze the fairness principles of this formulation, let us consider a situation where the community lacks of generation and faces an import cost $\gamma_{\text {imp }} q_{\text {imp. At optimality, }}$ (1d) is satisfied with shadow price equal to $\gamma_{\text {imp }}$. It follows that

$$
\gamma_{\text {imp }} q_{\text {imp }}=\gamma_{\text {imp }} \sum_{j=1}^{n} \alpha_{j}
$$

Equation (7) shows how proportional fairness is intrinsic in the proposed formulation. The total costs are shared among the players and each prosumer $j$ faces a cost proportional to its contribution $\alpha_{j}$ to the total import.

\section{Penalty on the maximum importer}

Collaborative systems are prone to unfair behaviours, whenever one or more participants behave strategically. The community manager has the task to preserve fairness inside the collective. Inheriting demand charges mechanisms, load shape related features can be regulated. In this framework, min-max protocols are useful techniques for the community manager to minimize any maximum "flow".

One of the objectives of this work is to demonstrate that fairness among collective participants can be included in the distributed negotiation mechanisms. For instance, to prevent strategic behaviour, a community may choose to penalize the prosumer contributing the most to the import by means of an additional fee. Each member is, therefore, pushed to decrease its import, as this fee increases. We model this by adding a penalization $\gamma_{\max }$ to the maximum component of prosumers import shares (corresponding to the $l_{\infty}$ norm when all vector components are non-negative). The function $g$ then becomes

$$
g\left(q_{\text {imp }}, q_{\exp }, \boldsymbol{\alpha}\right)=\gamma_{\text {imp }} q_{\text {imp }}+\gamma_{\exp } q_{\exp }+\gamma_{\max }\|\boldsymbol{\alpha}\|_{\infty}
$$

where $\boldsymbol{\alpha}=\left(\alpha_{1}, \ldots, \alpha_{n}\right)$. The resulting problem preserves convexity, since the $l_{\infty}$ norm of a vector is a convex function. However, as the $l_{\infty}$ norm needs all the components $\alpha_{j}$ to be calculated, the problem cannot be decomposed anymore. To maintain a decomposable structure, we rewrite (8) as

$$
\begin{gathered}
g\left(q_{\text {imp }}, q_{\exp }, \vartheta\right)=\gamma_{\text {imp }} q_{\text {imp }}+\gamma_{\exp } q_{\exp }+\gamma_{\max } \vartheta \\
\text { with } \quad \alpha_{j} \leq \vartheta, \quad j=1, \ldots, n
\end{gathered}
$$

In this way, $g$ is expressed as function of the upper bound, $\vartheta$, of the $l_{\infty}$ norm, also known as epigraph formulation, and the inequality constraints grant to achieve the same solution and to allow for decomposition techniques.

\section{Peak-shaving services}

Minimax principles become useful not only to avoid strategic behaviours but also to implement interesting interface models. For instance, let us assume a multi-temporal optimization for an energy collective. The community manager can coordinate the prosumers to provide peak shaving services by minimizing the maximum imported energy over the considered timestamps (indexed with $t=1, \ldots, \mathcal{T}$ ), as in [18]. The definition of $g$ becomes

$$
g\left(\boldsymbol{q}_{\text {imp }}, \boldsymbol{q}_{\exp }\right)=\boldsymbol{\gamma}_{\text {imp }}^{T} \boldsymbol{q}_{\text {imp }}+\boldsymbol{\gamma}_{\exp }^{T} \boldsymbol{q}_{\text {exp }}+\gamma_{\text {peak }}\left\|\boldsymbol{q}_{\text {imp }}\right\|_{\infty}
$$

where all the bold variables and parameter are to be intended as vectors of size $\mathcal{T}$ and $\gamma_{\text {peak }}$ the penalization coefficient. Unlike (8), (10) preserves the decomposable structure of the optimization problem. To simplify the problem solution, the $l_{\infty}$ norm can still be linearized by means of the upper bound of $q_{\text {imp,t }}$ for all $t=1, \ldots, \mathcal{T}$. It follows that

$$
\begin{aligned}
& g\left(\boldsymbol{q}_{\text {imp }}, \boldsymbol{q}_{\text {exp }}, \vartheta\right)=\boldsymbol{\gamma}_{\text {imp }}^{T} \boldsymbol{q}_{\text {imp }}+\boldsymbol{\gamma}_{\text {exp }}^{T} \boldsymbol{q}_{\text {exp }}+\gamma_{\text {peak }} \vartheta \\
& \text { with } \quad q_{i m p, t} \leq \vartheta, \quad \forall t
\end{aligned}
$$


Fairness of these formulation is granted in terms of minimax principles. The energy "flows" addressed are flattened more, the higher the penalization coefficient. This does not imply that the system preserves fairness under other definitions, e.g. variation of price or proportional total costs. The system fairness is strictly related to the criteria the collective members agree to adopt.

\section{E. Geographical preferences}

Energy collectives are to be envisaged as a market construct and not related to a certain positioning on the electric power grid. In practice, this means that operations on a micro-grid, both off- and connected to the grid [6], is only one possible configuration, but more generally such communities may be formed irrespective of grid connection and geographical location. The use of distribution (or transmission for longer distances) networks for community trades results in additional grid costs including several parameters, e.g. geographical distance and lines congestion.

We include these additional costs by investigating a setup where an energy collective is formed by prosumers from three different neighbourhoods, $N_{1}, N_{2}, N_{3}$. We model this system by splitting (1c) in three sub-communities and by defining energy flows, $\boldsymbol{v}=\left(v_{12}, v_{13}, v_{23}\right)$, on each line connecting the neighbourhoods, as depicted in Figure 2. We include geographical differentiation as relative transaction costs $\gamma_{\text {geo }}=$ $\left(\gamma_{\text {geo, } 12}, \gamma_{\text {geo, } 13}, \gamma_{\text {geo, 23 }}\right)$. It follows that

$$
\begin{aligned}
g\left(q_{\text {imp }}, q_{\text {exp }}\right) & =\gamma_{\text {imp }} q_{\text {imp }}+\gamma_{\exp } q_{\text {exp }}+\gamma_{\text {geo }}^{T}|\boldsymbol{v}| \\
\sum_{j \in N_{1}} q_{j} & =+v_{12}+v_{13} \\
\sum_{j \in N_{2}} q_{j} & =-v_{12}+v_{23} \\
\sum_{j \in N_{3}} q_{j} & =-v_{13}-v_{13}
\end{aligned}
$$

This formulation allows to represent technical constraints, typical of power flow analysis, in the form of spatial and temporal varying grid tariffs. This becomes fundamental in view of a possible integration of energy collectives in the current system operation. Furthermore, the collective can be fragmented to the extreme, i.e. to single prosumer, accounting for individual trades, as in [19]. In this way, the energy collective becomes a supervised version of a fully peer-to-peer economic dispatch.

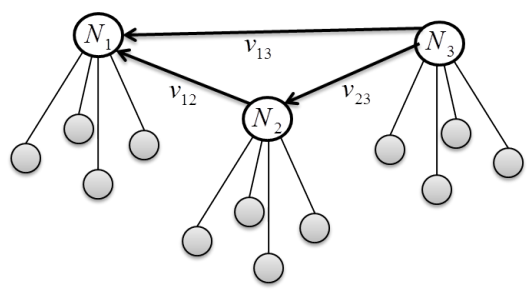

Fig. 2. Energy fluxes between 3 neighbourhoods.

\section{Distributed Optimization APPROACH TO THE OPERATION OF ENERGY COLLECTIVES}

The nature of (1) readily suggests employing decomposition techniques to achieve optimality in a distributed fashion. More precisely, the Alternating Direction Method of Multipliers (ADMM [20]) is used here. This algorithm allows to explicitly define individual problems for each prosumer and supervise the exchange of information between the collective members and the community manager. Alternative decomposition approaches could be employed, with their advantages and caveats, as recently reviewed and compared in [21] for optimal power flow problems. Langragian Relaxation (LR) and Proximal Message Passing (PMP) are valuable alternative solving algorithms for the market structure proposed. We find that ADMM naturally fits the market structure of energy collectives, as it entails a supervisory node (not required for instance with PMP). In addition, ADMM generalizes LR, as it does not require the objective function to be smooth and differentiable, limiting the requirements to impose to the community members.

\section{A. Problem formulation}

In order to decompose the community economic dispatch with ADMM, we rewrite (1) in terms of (3). It follows that

$$
\begin{array}{ll}
\min _{\Gamma} & \sum_{j=1}^{n} \tilde{f}_{j}\left(p_{j}, q_{j}, \alpha_{j}, \beta_{j}\right)+g\left(q_{\text {imp }}, q_{\text {exp }}\right) \\
\text { s.t. } & \sum_{j=1}^{n} q_{j}=0 \\
& \sum_{j=1}^{n} \alpha_{j}=q_{\text {imp }} \\
& \sum_{j=1}^{n} \beta_{j}=q_{\text {exp }}
\end{array}
$$

where the only explicit constraints are the coupling constraints among prosumers sub-problems. In case of coupling inequality constraints, for instance when $g$ has the form of (9), additional variables $\delta_{j}$ are used as upper bounds for the variables of the sub-problems. In this case (9) becomes

$$
\begin{array}{ll}
\min _{\Gamma} & \sum_{j=1}^{n} \tilde{f}_{j}\left(p_{j}, q_{j}, \alpha_{j}, \beta_{j}, \delta_{j}\right)+g\left(q_{\text {imp }}, q_{\text {exp }}, \vartheta\right) \\
\text { s.t. } & \sum_{j=1}^{n} q_{j}=0 \\
& \sum_{j=1}^{n} \alpha_{j}=q_{\text {imp }} \\
& \sum_{j=1}^{n} \beta_{j}=q_{\text {exp }} \\
& \delta_{j}=\vartheta, \quad j=1, \ldots, n
\end{array}
$$


with the feasible set $\mathscr{R}_{j}$ of each function $\tilde{f}_{j}$ including the additional constraint $\alpha_{j} \leq \delta_{j}$. We can now write the augmented Lagrangian, for instance of (14), as

$$
\begin{aligned}
L_{\rho}(x, z, y)= & \sum_{j=1}^{n} \tilde{f}_{j}\left(x_{j}\right)+\tilde{g}(z)+ \\
& +y^{T}(A x+B z)+\frac{\rho}{2}\|A x+B z\|_{2}^{2}
\end{aligned}
$$

where, $\quad x_{j}=\left(p_{j}, q_{j}, \alpha_{j}, \beta_{j}, \delta_{j}\right) \quad z=\left(q_{\text {imp }}, q_{\text {exp }}, \vartheta\right)$

$$
\begin{aligned}
x & =\left(q_{1}, \ldots, q_{n}, \alpha_{1}, \ldots, \alpha_{n}, \beta_{1}, \ldots, \beta_{n}, \delta_{1}, \ldots, \delta_{n}\right) \\
A & =\left[\begin{array}{cccc}
\mathbf{1}^{T} & \mathbf{0}^{T} & \mathbf{0}^{T} & \mathbf{0}^{T} \\
\mathbf{0}^{T} & \mathbf{1}^{T} & \mathbf{0}^{T} & \mathbf{0}^{T} \\
\mathbf{0}^{T} & \mathbf{0}^{T} & \mathbf{1}^{T} & \mathbf{0}^{T} \\
0 & \mathbb{0} & \mathbb{0} & I
\end{array}\right] \quad B=\left[\begin{array}{ccc}
0 & 0 & 0 \\
-1 & 0 & 0 \\
0 & -1 & 0 \\
\mathbf{0} & \mathbf{0} & -\mathbf{1}
\end{array}\right]
\end{aligned}
$$

with 0 and $1 n$-dimensional vectors of zeros and ones, and 0 and $I$ the $n$-dimensional null and identity matrices. The optimization algorithm follows the common ADMM steps

$$
\begin{aligned}
x_{j}^{k+1} & =\underset{x_{j}}{\arg \min } L_{\rho}\left(x_{j}, z^{k}, y^{k}\right), \quad j=1, \ldots, n \\
z^{k+1} & =\underset{z}{\arg \min } L_{\rho}\left(x^{k+1}, z, y^{k}\right) \\
y^{k+1} & =y^{k}+\rho\left(A x^{k+1}+B z^{k+1}\right)
\end{aligned}
$$

Convergence is monitored, at each iteration $k$, via the primal residual $r^{k+1}=A x^{k+1}+B z^{k+1}$ and the dual residual $s^{k+1}=\rho A^{T} B\left(z^{k+1}-z^{k}\right)$. Whenever $\left\|r^{k+1}\right\|_{2}$ and $\left\|s^{k+1}\right\|_{2}$ are smaller than the desired tolerance, the stopping criterion is met.

\section{B. Convergence analysis}

ADMM formulation ensures that, whenever the optimization problem is closed, proper and convex, the algorithm converges to optimality [20]. However when operating with real data, numerical issues challenge convergence within a reasonable number of iterations. In particular, large oscillations of the Lagrangian multiplier $y$ relative to (14b) mine convergence of ADMM. These oscillations occur as the residual of (14b) has no direct link to any global variable (first line of matrix $B$ of all zeros). We solve this numerical issue by weighting the constraint with a factor of $\frac{1}{n}$, hence considering not the whole residual but its average over the prosumers. In this way, the oscillations are smoothed out and the method converges more easily.

A similar issue occurs when we apply ADMM to clear a market with function $g$ as in (11). The penalization for the maximum import happens only on the global problem $(z$-update) and has no direct link to the $x$-update. We notice that when $\vartheta$ has non-zero values, the Lagrangian multiplier of the import balance increases of the penalization factor, leading to large convergence oscillations. We rewrite (11) as $\sum_{j=1}^{n} \delta_{j, t} \leq \vartheta$ and no more as function of $q_{\text {imp. }}$. In this way, the Lagrangian multiplier of the import balance remains the import price and the penalization is directly mirrored at a community level. Oscillations are again smoothed helping the algorithm to reach convergence.
One more numerical issue arises as we find ADMM sensitive to its penalization parameter $\rho$. Boyd et al. proved convergence of ADMM for any $\rho$ value in [20], however the number of iterations needed to achieve optimality can largely exceed operational requirements. For this reason, we bound $10^{-5}<\rho<1$ and adopt an heuristic adapting scheme based on a moving window, $W$, of the residuals norm, $\left\|r_{k}\right\|_{2}$. We update $\rho$ at each iteration as

$$
\rho^{k}= \begin{cases}2 \rho^{k-1} & \max _{j=1, \ldots, W}\left|\left(\left\|r_{k}\right\|_{2}-\left\|r_{k-j}\right\|_{2}\right)\right| \leq 0.01\left\|r_{k}\right\|_{2} \\ \frac{\rho^{k-1}}{3} & \left\|r_{k}\right\|_{2}-\frac{\sum_{j=1}^{W}\left\|r_{k-j}\right\|_{2}}{W} \geq 0.1\left\|r_{k}\right\|_{2} \\ \rho^{k-1} & \text { otherwise }\end{cases}
$$

The penalization factor, $\rho$, is reduced with a factor of 3 whenever the primal residual is $10 \%$ higher than the average on its past values (within the considered window). This allows to detect oscillations of the algorithm and smooth them by reducing the update step of the Lagrangian multipliers. Whenever $\left\|r_{k}\right\|_{2}$ changes less of $1 \%$ along the moving window, $\rho$ is doubled. Hence, the Lagrangian multipliers are updated with a larger step and convergence is speeded up.

ADMM provides a general method that grants convergence independently from the nature of the optimization problem. As we find ADMM to be sensitive to numerical properties of the dataset, application-specific methods may achieve better performances. However, we continue with ADMM, since relatively easy heuristic schemes solve the convergence issues for most of the simulations of this paper. Furthermore, the focus of this work relies more on the market structure and operational framework of energy collectives rather than on its solving methodology.

\section{Application and Case-studies}

As a basis for illustration and discussion, we consider a setup of 15 prosumers, most of them equipped with solar power generation and controllable load. The data was originally collected from households in Australia between July 2012 and June 2013 and shared as open data [22]. From the raw data, we extract the time series of fixed and flexible
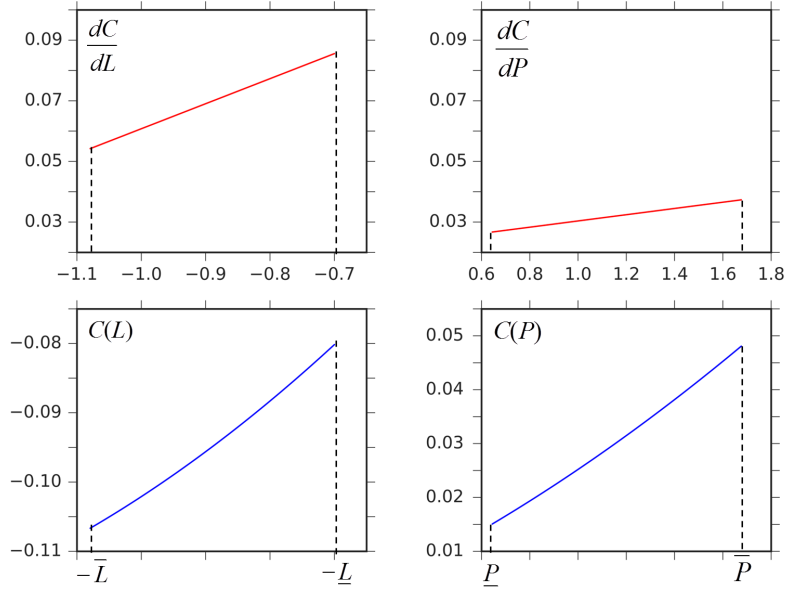

Fig. 3. Examples of cost curves (bottom) and marginal curves (top) for a consumer (left) and a generator (right) employed in our simulations. 
load, the latter corresponding to the energy consumed by controllable hot water tanks. Since the modelling of controllable loads is out of the scope of this work, we adopt an arbitrary load management system as following. We calculate the daily capacity of flexible consumption $\left(L^{*}\right)$ and we assume that, at each hour, the flexible content of each actor is twice this capacity. In this way, the electricity consumption is lower bounded by the fixed load $(\underline{L})$ and can range within the flexibility budget according to the agent's preferences up to $\bar{L}=\underline{L}+2 L^{*}$, as displayed in Figure 3 (note that in the figure the consumption is conventionally assumed negative). Finally, we fix the cumulative daily energy consumption of each prosumer to match the data of the reference dataset. This time-linking constraint implies that the energy collective market is cleared over 24 hours with a single optimization problem. As for the generation, in addition to solar production, we add synthetic data for 6 conventional generators, in order to extend our analysis to a test case with possibility of dispatchable generation. Given the small size of the collective, we assume that the community trades energy as price-taker with the wholesale market. The market prices are extracted from the Australian Energy Market Operator website.

\section{A. Prosumers costs curves}

As the cost functions are dependent on the preferences of each prosumer, we build them based on the day-ahead market price as following. We consider different curves for each quarter of the day (00:00-06:00, 06:00-12:00, 12:00-18:00 and 18:00-24:00). The price $a$, that each prosumer is willing to pay (or be paid) for its average load (production) and the increase (or decrease) of price, $b$, corresponding to the minimum setpoint $\underline{U}_{i j}$ (or maximum $\bar{U}_{i j}$ ) of asset $i$, are sampled as

$$
a \sim \mathcal{N}\left(\mu_{D A}, \frac{\sigma_{D A}}{3}\right) \quad b \sim \mathcal{N}\left(0, \frac{\sigma_{D A}}{3}\right)
$$

The mean $\mu_{D A}$ and the standard deviation $\sigma_{D A}$ are calculated from the yearly wholesale market price in the same hours interval. In both cases we shrink the standard deviation of the market price to avoid negative values in the prosumers marginal curves. Assuming quadratic cost curves as in (2), their marginal functions are linear functions of the assets setpoint $u_{i j}$ and can be written as $c_{i j} u_{i j}+d_{i j}$, with

$$
c_{i j}=2 \frac{b}{\bar{U}_{i j}-\underline{U}_{i j}} \quad d=a+b \frac{\bar{U}_{i j}+\underline{U}_{i j}}{\bar{U}_{i j}-\underline{U}_{i j}}
$$

As we use negative values of $u_{i j}$ for energy consumption, $\underline{U}_{i j}$ corresponds to the reciprocal of the maximum load $-\bar{L}$. The same procedure is implemented for the cost curves of the generators, but we twist the mean of the market price to model three cheaper base-load generators and three more expensive peak generators. Figure 3 displays two of the curves employed in the simulated test-case.

\section{B. Simulations results}

As we simulate the energy collective market structure on the described dataset, we assume a transaction cost for the energy traded within the community of $\gamma_{\mathrm{com}}=1 \$ / \mathrm{MWh}$ and

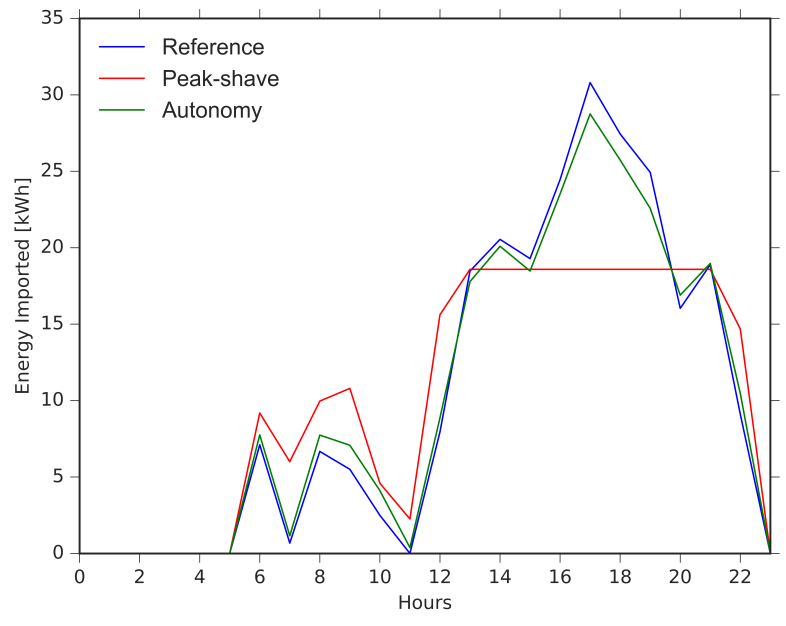

Fig. 4. Example of energy imported profiles under different interface models.

a spread between import and export price of $\tau=100 \$ / \mathrm{MWh}$. Each simulation is then solved with both a single optimization approach and by means of decomposition techniques, i.e. ADMM. Convergence is reached in most timestamps and whenever the iterative routine stops after reaching the maximum number of iterations allowed $\left(10^{4}\right)$, the optimality gap is negligible. We find that the maximum gap among all the simulations is around $0.1 \%$. The large number of iterations allowed and a low tolerance on the stopping criterion $\left(\varepsilon=10^{-6}\right)$ justifies these small optimality gaps. As real applications do not require such high precision, the number of iterations needed can be significantly decreased.

We use as benchmark an optimization model in which the prosumers trade individually in the wholesale market. Results show that the collective has a total cost of around $1600 \$$, compared to a total system cost of around $5250 \$$ in case of independent trading. Not only the community achieves lower costs over one year, but all prosumers are individually better off when allowed to exchange energy within the collective. All community trading models achieve lower yearly costs compared to the benchmark model, as in Table I. However, one should not conclude that prosumers always achieve lower system costs by joining an energy collective, since the main objective is to respect common agreements and preferences. For instance, in case of a community that strongly aims for autonomy, hence minimizing the import from the market operator with a large penalization coefficient, the internal price could increase, according to the participants' cost and utility curves, and result in higher total costs.

The main result, however, is that, it is only by changing the common agreements modeled by the community manager, that the prosumers behaviour, and consequently the system costs, are affected. An increase of $16 \%$ of the yearly costs occurs when considering geographical preferences, while the system costs are more than doubled when the community seeks autonomy from the system operator. This reflects the costs the community is willing to bear respectively for including geographical grid costs and for decreasing the volumes traded in the wholesale market of around $20 \%$ for imported and 
TABLE I

RESULTS AND FAIRNESS INDICATORS OF ALL SIMULATED MODELS.

\begin{tabular}{|c|c|c|c|c|c|c|c|c|c|}
\hline \multirow{2}{*}{$\begin{array}{l}\text { TRADING MODEL } \\
\text { Interface model }\end{array}$} & \multicolumn{3}{|c|}{ SiMULATIONS RESULTS } & \multicolumn{2}{|c|}{ QoS } & \multicolumn{2}{|c|}{ QoE } & \multicolumn{2}{|c|}{ MiM } \\
\hline & Total costs $[\$]$ & Total import [MWh] & Total export $[\mathrm{MWh}]$ & Mean & St. dev. & Mean & St. dev. & Mean & St. dev. \\
\hline INDIVIDUAL TRADING & 5245.30 & 55.52 & 45.49 & - & - & 0.15 & 0.089 & 0.00 & 0.001 \\
\hline \multicolumn{10}{|l|}{ COMMUNITY TRADING } \\
\hline Market-based & 1577.43 & 16.71 & 11.77 & 0.54 & 0.096 & 0.95 & 0.112 & 0.00 & 0.005 \\
\hline Autonomy & 4064.28 & 13.78 & 5.44 & 0.56 & 0.101 & 0.91 & 0.198 & 0.00 & 0.004 \\
\hline Min-max import share & 1568.28 & 16.60 & 11.75 & 0.57 & 0.077 & 0.45 & 6.231 & 1.00 & 0.020 \\
\hline Peak-shaving & 1626.01 & 16.65 & 11.73 & 0.53 & 0.114 & 0.93 & 0.155 & 0.01 & 0.058 \\
\hline Geographical & 1836.93 & 18.17 & 13.37 & 0.52 & 0.099 & 0.81 & 0.137 & 0.00 & 0.003 \\
\hline
\end{tabular}

$50 \%$ for exported energy. The community manager impacts the behaviour of the prosumers also in terms of energy dispatch. Figure 4 displays how the community aggregated load, i.e. the energy imported, is flattened and its peak reduced, in this case of around $30 \%$, when including peak-shaving mechanisms.

\section{Fairness indicators}

As we show how the community manager can impact prosumers behaviour, we analyze the relationship of this influence among the single community members. To do this, we refer to fairness indicators normally used in communication networks, specifically estimating Quality of Service (QoS), Quality of Experience (QoE) and Min-Max fairness (MiM). QoS indicators are normally used to assess allocation fairness by means of Jain's index [23], defined as

$$
\mathrm{QoS}=\frac{\left[\sum_{j=1}^{n}\left|q_{j}\right|\right]^{2}}{\sum_{j=1}^{n} q_{j}^{2}}
$$

We calculate this indicator on the volumes of energy traded within the community. Under this criterion, the system is $100 \%$ fair when the energy volumes traded in the collective are equal for all players. Equal volumes traded inside the community is not necessarily an interesting outcome for this market structure. However, low values of QoS can spot the presence of players with larger impact in the community, that may mine the robustness of the collective towards strategic behaviours. Results in Table I show that the community is around $55 \%$ fair in terms of participation in community trades. The different capacity among community members justifies the relatively low QoS index. We find the volumes of the community trades stable among the simulated models of the community manager. A lower QoS index is calculated for the market including geographical preferences, as they act as economical bottlenecks within the community and impact the energy flows among prosumers in the form of increased trades inside neighbourhoods.

Quality of Service indicators allow to evaluate market disequilibrium in terms of the service considered. To move away from a system perspective towards a user-centric point of view, we calculate a second index to evaluate fairness of consumers satisfaction, namely Quality of Experience. We base consumers satisfaction on the perceived price of energy

$$
\lambda_{j}=\frac{\lambda_{\mathrm{DA}} \beta_{j}-\left(\lambda_{\mathrm{DA}}+\tau\right) \alpha_{j}-\lambda_{\mathrm{com}} q_{j}}{p_{j}+l_{j}}
$$

We calculate $\lambda_{j}$ for each prosumer as the sum of costs or revenues from trading within the community and with the system operator divided by the net power consumed or produced. We assess fairness from users perspective, by means of the index proposed in [24], as

$$
\mathrm{QoE}=1-\frac{\sigma}{\sigma_{\max }}
$$

where $\sigma$ is the standard deviation of prices $\lambda_{j}$ and $\sigma_{\max }=$ $\lambda_{\text {imp }}-\lambda_{\text {exp }}$ the maximum price deviation. Hence, the energy collective fairness is higher as the price variation among the prosumers is smaller, with maximum $\mathrm{QoE}=1$ whenever all prosumers prices $\lambda_{j}$ are the same. Our simulations show that fairness increases, from 0.15 to 0.95 , as the prosumers move from an individual to a community-based market framework.

The impact of community manager models is considerable, especially when the maximum importer is penalized. In this case, the yearly mean of the QoE indicator is around 0.45 with a large standard deviation, 6.2. This happens because the large penalization factor employed pushes all the import shares of the collective members to the same value. We verify this by computing the Min-Max indicator,

$$
\operatorname{MiM}=\frac{\min \alpha_{j}}{\max \alpha_{j}}
$$

The index achieves a value of 1.0, meaning that the import shares are almost always equal among prosumers (standard deviation of 0.02). This implies that also prosumers with no need for energy import are forced to buy their share from the system operator and sell it to others community members. This leads to larger spread of the individually perceived prices, i.e. to a lower system fairness.

The results so far discussed consider a fixed penalization coefficient on the maximum importer of $\gamma_{\max }=1 \$ / \mathrm{MWh}$. As displayed in Figure 5, where $\gamma_{\max }$ varies from $0 \$ / \mathrm{MWh}$, as in the market-based community trading case, and $1 \$ / \mathrm{MWh}$, the impact of common agreements on the investigated fairness indicators changes for different penalty factors. This result justifies our claim to design a market organization in which 


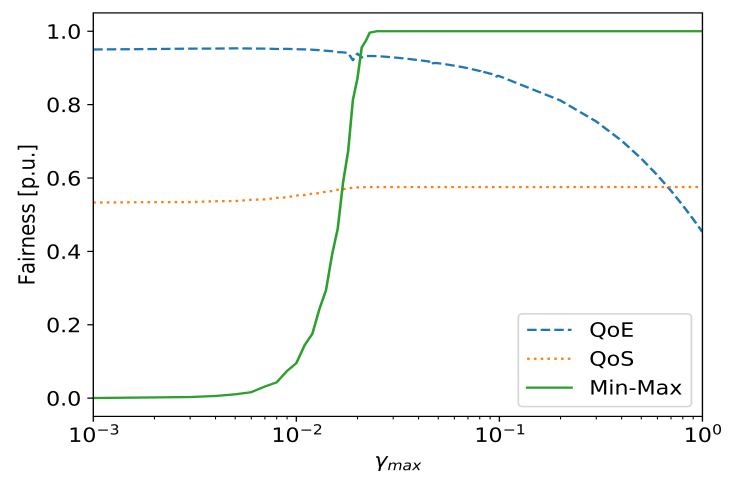

Fig. 5. Impact of coefficient $\gamma_{\max }$ on system fairness indicators.

the collective members are able to actively modify their energy procurement while preserving fairness according to their common agreements. It is, however, fundamental to underline that the degree of modification of the participants' energy procurement largely depends on their available flexibility as well as on their preferences and technology mix.

\section{CONCLusions}

Acknowledging the increasing installed capacity of DERs, we proposed the energy collective as a new community-based market structure, that allows prosumers to actively optimize their assets. We showed that a distributed market structure achieves optimal economic dispatch for the community and that different collective agreements, granted by a third-party node, influence the negotiation process. This supervisory node, i.e. community manager, directly impacts the prosumers in terms of energy dispatch as well as revenues and payments. We evaluated this by means of fairness indicators, typical of telecommunication systems and adapted to our case study.

The proposed market structure for energy collectives defines a general framework easy to adapt for further studies. We foresee scalability as the first challenge when dealing with consumer-centric markets. Additional work needs to be done to compare community-based, peer-to-peer and hybrid market organizations when the number of players increases. Particular attention will be paid to fairness among prosumers and to the clearing algorithms, especially to their performances in the more realistic case of asynchronous communication. After investigating the robustness of consumer-centric market structures towards larger and more realistic test cases, we plan to focus our research on the integration of these new market constructs within system operations. Price-maker and strategic behaviours will be considered as well as the design of new dynamic grid tariffs to directly reflect grid costs in the market mechanisms.

\section{REFERENCES}

[1] C. Eid, P. Codani, Y. Perez, J. Reneses, and R. Hakvoort, "Managing electric flexibility from distributed energy resources: A review of incentives for market design," Renewable and Sustainable Energy Reviews, vol. 64, pp. 237-247, 2016.

[2] P. Palensky and D. Dietrich, "Demand side management: Demand response, intelligent energy systems, and smart loads," IEEE Transactions on Industrial Informatics, vol. 7, no. 3, pp. 381-388, 2011.
[3] S. M. Nosratabadi, R. A. Hooshmand, and E. Gholipour, "A comprehensive review on microgrid and virtual power plant concepts employed for distributed energy resources scheduling in power systems," Renewable and Sustainable Energy Reviews, vol. 67, pp. 341-363, 2017.

[4] S. Burger, J. P. Chaves-Ávila, C. Batlle, and I. J. Pérez-Arriaga, "A review of the value of aggregators in electricity systems," Renewable and Sustainable Energy Reviews, vol. 77, pp. 395-405, 2017.

[5] T. Van Der Schoor and B. Scholtens, "Power to the people: Local community initiatives and the transition to sustainable energy," Renewable and Sustainable Energy Reviews, vol. 43, pp. 666-675, 2015.

[6] Y. Parag and B. K. Sovacool, "Electricity market design for the prosumer era," Nature Energy, vol. 1, no. 4, p. 16032, 2016.

[7] C. Giotitsas, A. Pazaitis, and V. Kostakis, "A peer-to-peer approach to energy production," Technology in Society, vol. 42, pp. 28-38, 2015.

[8] J. Qin, R. Rajagopal, and P. P. Varaiya, "Flexible market for smart grid: coordinated trading of contingent contracts," IEEE Transactions on Control of Network Systems, 2017.

[9] S. Rusitschka, K. Eger, and C. Gerdes, "Smart grid data cloud: A model for utilizing cloud computing in the smart grid domain," in 2010 First IEEE International Conference on Smart Grid Communications (SmartGridComm). IEEE, 2010, pp. 483-488.

[10] M. Mihaylov, S. Jurado, N. Avellana, K. Van Moffaert, I. M. de Abril, and A. Nowé, "Nrgcoin: Virtual currency for trading of renewable energy in smart grids," in 2014 11th International Conference on the European Energy Market (EEM). IEEE, 2014, pp. 1-6.

[11] S. Chen and C. C. Liu, "From demand response to transactive energy: state of the art," Journal of Modern Power Systems and Clean Energy, vol. 5, no. 1, pp. 10-19, 2017.

[12] B. P. Koirala, E. Koliou, J. Friege, R. A. Hakvoort, and P. M. Herder, "Energetic communities for community energy: A review of key issues and trends shaping integrated community energy systems," Renewable and Sustainable Energy Reviews, vol. 56, pp. 722-744, 2016.

[13] Y. Guo, M. Pan, Y. Fang, and P. P. Khargonekar, "Decentralized coordination of energy utilization for residential households in the smart grid," IEEE Transactions on Smart Grid, vol. 4, no. 3, pp. 1341-1350, 2013.

[14] G. Hug, S. Kar, and C. Wu, "Consensus + innovations approach for distributed multiagent coordination in a microgrid," IEEE Transactions on Smart Grid, vol. 6, no. 4, pp. 1893-1903, 2015.

[15] R. Verschae, T. Kato, and T. Matsuyama, "Energy management in prosumer communities: A coordinated approach," Energies, vol. 9, no. 7, p. 562, 2016.

[16] N. Liu, X. Yu, C. Wang, C. Li, L. Ma, and J. Lei, "Energy-sharing model with price-based demand response for microgrids of peer-to-peer prosumers," IEEE Transactions on Power Systems, vol. 32, no. 5, pp. 3569-3583, 2017.

[17] R. Carvalho, L. Buzna, R. Gibbens, and F. Kelly, "Critical behaviour in charging of electric vehicles," New Journal of Physics, vol. 17, no. 9, p. 095001, 2015.

[18] I. Notarnicola, M. Franceschelli, and G. Notarstefano, "A dualitybased approach for distributed min-max optimization with application to demand side management," in 2016 IEEE 55th Conference on Decision and Control (CDC). IEEE, 2016, pp. 1877-1882.

[19] E. Sorin, L. A. Bobo, and P. Pinson, "Consensus-based approach to peer-to-peer electricity markets with product differentiation," IEEE Transaction on Power Systems, 2018, working paper.

[20] S. Boyd, N. Parikh, E. Chu, B. Peleato, J. Eckstein et al., "Distributed optimization and statistical learning via the alternating direction method of multipliers," Foundations and Trends in Machine Learning, vol. 3, no. 1, pp. 1-122, 2011.

[21] A. Kargarian, J. Mohammadi, J. Guo, S. Chakrabarti, M. Barati, G. Hug, S. Kar, and R. Baldick, "Toward distributed/decentralized dc optimal power flow implementation in future electric power systems," IEEE Transactions on Smart Grid, 2016.

[22] E. L. Ratnam, S. R. Weller, C. M. Kellett, and A. T. Murray, "Residential load and rooftop pv generation: an australian distribution network dataset," International Journal of Sustainable Energy, vol. 36, no. 8, pp. 787-806, 2017.

[23] R. Jain, D.-M. Chiu, and W. R. Hawe, A quantitative measure of fairness and discrimination for resource allocation in shared computer system. Eastern Research Laboratory, Digital Equipment Corporation Hudson, MA, 1984, vol. 38 .

[24] T. Hoßfeld, L. Skorin-Kapov, P. E. Heegaard, and M. Varela, "Definition of qoe fairness in shared systems," IEEE Communications Letters, vol. 21, no. 1, pp. 184-187, 2017. 


\section{APPENDIX}

In this appendix we demonstrate market efficiency for the proposed energy collectives. Our approach is to show that the energy collective market, modelled as a centralized optimization problem, solves exactly the same problem as an equilibrium problem in which every agent optimizes its energy procurement while subjected to market constraints. In practice, this is done by checking that the KKT conditions of both problems coincide. We start by considering the extended version of (1) as

$$
\begin{aligned}
& \min _{\Gamma} \sum_{j=1}^{n}\left[\sum_{i \in I_{j}} \psi_{i j}\left(u_{i j}\right)+h_{j}\left(q_{j}, \alpha_{j}, \beta_{j}\right)\right] \\
& +g\left(q_{\text {imp }}, q_{\text {imp }}\right) \\
& \text { s.t. } \quad \sum_{i \in I_{j}} u_{i j}+q_{j}+\alpha_{j}+\beta_{j}=0 \quad \forall j \quad\left(\pi_{j}\right) \\
& \sum_{j=1}^{n} q_{j}=0 \\
& \sum_{j=1}^{n} \alpha_{j}-q_{\text {imp }}=0 \\
& \sum_{j=1}^{n} \beta_{j}-q_{\exp }=0 \\
& u_{i j}-\bar{U}_{i j} \leq 0 \\
& \underline{U}_{i j}-u_{i j} \leq 0 \\
& -\alpha_{j} \leq 0 \\
& -\beta_{j} \leq 0 \\
& -q_{\text {imp }} \leq 0 \\
& -q_{\exp } \leq 0 \\
& \forall j \quad\left(\gamma_{j}^{a}\right) \\
& \forall j \quad\left(\gamma_{j}^{b}\right)
\end{aligned}
$$

(16a)

where the dual variables of each constraint are reported within brackets. The KKT conditions of (16) then become

$$
\begin{array}{ll}
\psi_{i j}^{\prime}\left(u_{i j}\right)+\pi_{j}+\gamma_{i j}^{M}-\gamma_{i j}^{m}=0 & \forall i, j \\
\frac{\partial h}{\partial q_{j}}+\pi_{j}+\lambda_{c}=0 & \forall j \\
\frac{\partial h}{\partial \alpha_{j}}+\pi_{j}+\lambda_{i}-\gamma_{j}^{a}=0 & \forall j \\
\frac{\partial h}{\partial \beta_{j}}+\pi_{j}+\lambda_{e}-\gamma_{j}^{b}=0 & \forall j \\
\frac{\partial g}{\partial q_{\text {imp }}}-\lambda_{i}+\gamma_{i}=0 & \\
\frac{\partial g}{\partial q_{\text {exp }}}-\lambda_{e}+\gamma_{e}=0 & \\
\sum_{i \in I_{j}} u_{i j}+q_{j}+\alpha_{j}+\beta_{j}=0 & \forall j \\
\sum_{j=1}^{n} q_{j}=0 & \\
\sum_{j=1}^{n} \alpha_{j}-q_{\text {imp }}=0 &
\end{array}
$$

$$
\begin{array}{ll}
\sum_{j=1}^{n} \beta_{j}-q_{\exp }=0 & \\
0 \leq \bar{U}_{i j}-u_{i j} \perp \gamma_{i j}^{M} \geq 0 & \forall i, j \\
0 \leq u_{i j}-\underline{U}_{i j} \perp \gamma_{i j}^{m} \geq 0 & \forall i, j \\
0 \leq \alpha_{j} \perp \gamma_{j}^{a} \geq 0 & \forall j \\
0 \leq \beta_{j} \perp \gamma_{j}^{b} \geq 0 & \forall j \\
0 \leq q_{\text {imp }} \perp \gamma_{i} \geq 0 & \\
0 \leq q_{\text {exp }} \perp \gamma_{e} \geq 0 &
\end{array}
$$

We then write the relative equilibrium problem, in which each prosumer $j$ minimizes its costs following

$$
\begin{array}{llr}
\min _{\Gamma} & \sum_{i \in I_{j}} \psi_{i j}\left(u_{i j}\right)+h_{j}\left(q_{j}, \alpha_{j}, \beta_{j}\right)+ & \\
& \lambda_{c} q_{j}+\lambda_{i} \alpha_{j}-\lambda_{e} \beta_{j} & \\
\text { s.t. } & \sum_{i \in I_{j}} u_{i j}+q_{j}+\alpha_{j}+\beta_{j}=0 & \left(\pi_{j}\right) \\
& u_{i j}-\bar{U}_{i j} \leq 0 \quad \forall i & \left(\gamma_{i j}^{M}\right) \\
& \underline{U}_{i j}-u_{i j} \leq 0 \quad \forall i & \left(\gamma_{i j}^{m}\right) \\
& -\alpha_{j} \leq 0 & \left(\gamma_{j}^{a}\right) \\
& -\beta_{j} \leq 0 & \left(\gamma_{j}^{b}\right)
\end{array}
$$

where $\lambda_{c}$ is the price for trading within the community and $\lambda_{i}$ and $\lambda_{e}$ respectively the price for buying and selling energy with the market operator. At the same time, the community manager, since non-profit, aims for the minimization of the costs related to internal common agreements and interface with market or system operator. It follows that

$$
\begin{array}{cll}
\min _{\Gamma} & g\left(q_{\text {imp }}, q_{\text {imp }}\right) & \\
\text { s.t. } & -q_{\text {imp }} \leq 0 & \left(\gamma_{i}\right) \\
& -q_{\text {exp }} \leq 0 & \left(\gamma_{e}\right)
\end{array}
$$

Under the assumption of perfect competition, we can model market constraints as

$$
\begin{array}{ll}
\sum_{j=1}^{n} q_{j}=0 & \left(\lambda_{c}\right) \\
\sum_{j=1}^{n} \alpha_{j}-q_{\text {imp }}=0 & \left(\lambda_{i}\right) \\
\sum_{j=1}^{n} \beta_{j}-q_{\exp }=0 & \left(\lambda_{e}\right)
\end{array}
$$

The KKT conditions of the equilibrium problem (18)-(20) become

$$
\begin{array}{ll}
\psi_{i j}^{\prime}\left(u_{i j}\right)+\pi_{j}+\gamma_{i j}^{M}-\gamma_{i j}^{m}=0 & \forall i, j \\
\frac{\partial h}{\partial q_{j}}+\pi_{j}+\lambda_{c}=0 & \forall j \\
\frac{\partial h}{\partial \alpha_{j}}+\pi_{j}+\lambda_{i}-\gamma_{j}^{a}=0 & \forall j \\
\frac{\partial h}{\partial \beta_{j}}+\pi_{j}+\lambda_{e}-\gamma_{j}^{b}=0 & \forall j
\end{array}
$$




$$
\begin{array}{ll}
\sum_{i \in I_{j}} u_{i j}+q_{j}+\alpha_{j}+\beta_{j}=0 & \forall j \\
0 \leq \bar{U}_{i j}-u_{i j} \perp \gamma_{i j}^{M} \geq 0 & \forall i, j \\
0 \leq u_{i j}-\underline{U}_{i j} \perp \gamma_{i j}^{m} \geq 0 & \forall i, j \\
0 \leq \alpha_{j} \perp \gamma_{j}^{a} \geq 0 & \forall j \\
0 \leq \beta_{j} \perp \gamma_{j}^{b} \geq 0 & \forall j \\
\frac{\partial g}{\partial q_{\text {imp }}}-\lambda_{i}+\gamma_{i}=0 & \\
\frac{\partial g}{\partial q_{\text {exp }}}-\lambda_{e}+\gamma_{e}=0 & \\
0 \leq q_{\text {imp }} \perp \gamma_{i} \geq 0 & \\
0 \leq q_{\text {exp }} \perp \gamma_{e} \geq 0 & \\
\sum_{j=1}^{n} q_{j}=0 & \\
\sum_{j=1}^{n} \alpha_{j}-q_{\text {imp }}=0 & \\
\sum_{j=1}^{n} \beta_{j}-q_{\text {exp }}=0 &
\end{array}
$$

As the two sets of KKT conditions, (17) and (21), are equal, this demonstrates that the optimization problem (16) for the market-based operation of energy collectives and the equilibrium problem (18)-(20) solve the same problem. This translates to ensuring market efficiency.

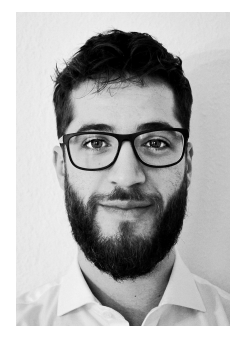

Fabio Moret received a M.Sc. degree in electrical engineering from the University of Padova, Padova, Italy, and a second M.Sc. degree in sustainable energy from the Technical University of Denmark, Kgs. Lyngby, Denmark. He is a Ph.D. student at the Technical University of Denmark, Centre for Electric Power and Energy, Department of Electrical Engineering. His research interests include consumer-centric electricity markets, distributed negotiation mechanisms, decomposition techniques for optimization problems and applied game theory.

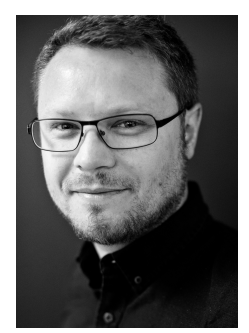

Pierre Pinson (SM'13) received the M.Sc. degree in applied mathematics from the National Institute for Applied Sciences, Toulouse, France, and the Ph.D. degree in energetics from Ecole des Mines de Paris, Paris, France. He is a Professor at the Department of Electrical Engineering, Centre for Electric Power and Energy, Technical University of Denmark, Kgs. Lyngby, Denmark, also heading a group focusing on Energy Analytics and Markets. His research interests include among others forecasting, uncertainty estimation, optimization under uncertainty, decision sciences, and renewable energies. He is an Editor for the International Journal of Forecasting and Wind Energy. 